\title{
Walter Höllerers Neuakzentuierung der Intellektuellenrolle im Literaturbetrieb
}

\author{
ROLF PARR
}

Im Folgenden wird der Frage nachgegangen, ob sich die Position von Walter Höllerer im Literaturbetrieb der 1960er Jahre mit Gewinn als die eines Intellektuellen beschreiben lässt, und ob eine solche Form der Beschreibung neue Sichtweisen auf das >Gesamtprojekt Höllerer` und en passant vielleicht auch den Literaturbetrieb seiner Zeit ermöglicht. Entsprechend geht es im ersten Teil darum, im Rückgriff auf Überlegungen der Interdiskurstheorie heuristisch zu bestimmen, was einen Intellektuellen eigentlich ausmacht. ${ }^{1}$ Der zweite Teil versucht auf dieser Basis dann die Aktivitäten Höllerers als ein Projekt der rotierend-integrativen Besetzung von Teilbereichen des Literatur-, Wissenschafts- und Medienbetriebs zu charakterisieren. Abschließend wird gefragt, warum dieses >Projekt Höllerer` im Grunde schon nach knapp zehn Jahren, nämlich mit der einsetzenden Studentenbewegung, als anachronistisch erscheinen musste.

1 Der bereits vielfach an anderer Stelle dargestellte Ansatz der Interdiskurstheorie soll hier nicht noch einmal ausführlich referiert werden. Die einschlägige Literatur ist verzeichnet in Parr, Rolf / Thiele, Matthias: Link(s). Eine Bibliografie zu den Konzepten `Interdiskurs`, >Kollektivsymbolik« und \Normalismus« sowie einigen weiteren Fluchtlinien. 2., stark erw. und überarb. Aufl., Heidelberg 2010. 
Was aber macht einen Intellektuellen eigentlich aus? Als konstitutive Merkmale sind zunächst einmal seine gesamtgesellschaftliche Sprecherrolle ${ }^{2}$ und damit verbunden ein gewisser »Anspruch auf Universalität« zu nennen. Georg Jäger hat den Intellektuellen daher dem Experten gegenübergestellt. »Der Experte« könne »sich auf fachspezifische Fähigkeiten und Kenntnisse berufen, die er in der Regel in einer Ausbildung erworben und durch Prüfungen nachgewiesen« habe. Demgegenüber seien »die Intellektuellen >Fachleute eines integrierenden Dilettantismus $\ll^{3}$ auf vielen Gebieten. Als »Spezialist[en] für das Wort $\ll^{4}$ schließen sie die verschiedenen gesellschaftlichen Teilbereiche sprachlich miteinander kurz. Wenn es aber offensichtlich um sprachliches Zusammenführen von Spezialwissensgebieten zu Synthesen geht, dann liegt es nahe, die Spezifik des Intellektuellen diskurstheoretisch zu reformulieren und ihn als >Spezialisten für diskursverbindende, inter-diskursive Synthesen zu charakterisieren, also als jemanden, der Breitenwirkung dadurch erzielt, dass er als nicht-spezieller Ort der Bündelung von im Alltag immer nur arbeitsteilig verfolgten Spezialgebieten fungiert und auf diese Weise vielfältig anschlussfähige und attraktive Publikumsprojekte in Form von imaginären Totalitäten anbietet. Genau dies haben Intellektuelle aber wiederum mit der ebenfalls hochgradig interdiskursiven modernen Literatur gemeinsam, sodass es nicht von ungefähr kommt, dass Schriftsteller vor Vertretern anderer Berufsgruppen wie Politikern und Wissenschaftlern immer wieder als Intellektuelle wahrgenommen und bezeichnet werden.

Die Position einer solchen integrativ wirkenden intellektuellen Stimme war nach dem Zweiten Weltkrieg jedoch vakant und für einige Zeit nur wechselnd besetzbar. ${ }^{5}$ Insbesondere schienen die als Interdiskursexperten dafür geradezu prädestinierten Schriftsteller nicht mehr

2 Vgl. Jäger, Georg: Der Schriftsteller als Intellektueller. In: Hanuschek, Sven / Hörnigk, Therese / Malende, Christine (Hg.): Schriftsteller als Intellektuelle. Politik und Literatur im Kalten Krieg. Tübingen 2000, S. 1-25.

3 Ebd., S. 6. Jäger zitiert hier Dircks, Walter: Heilige Allianz. Bemerkungen zur Diffamierung der Intellektuellen. In: Frankfurter Hefte (1961), S. 2332, hier S. 29.

4 Vgl. Jäger 2000, S. 3.

5 Für die »Gruppe 47« spricht Robert Neumann (Spezis. Gruppe 47 in Berlin. In: Konkret, 1966, H. 5, S. 34-39, hier 34) von »den Zufalls-Ausfüllern eines Vakuums«. 
infrage zu kommen, was zum einen daran lag, dass die NS-nahen Autoren viel vom positiven Kredit der Schriftsteller als hörenswerte öffentliche Stimmen verspielt hatten, zum anderen aber auch an einer generellen Skepsis gegenüber >großen Synthesen`, die stets auch Ideologien darstellen, ${ }^{6}$ was es schwer bis unmöglich machte, sie in der Nachkriegszeit wirkungsvoll ins Spiel zu bringen. Wollte man in dieser Situation dennoch die Position einer vom Literarischen herkommenden öffentlichen Stimme ausfüllen, dann musste dies völlig anders geschehen als durch die bisher üblichen Kopplungen im Diskursspektrum von Politik, Literatur, Religion und Populärwissenschaft. Andere Kombinationen gesellschaftlicher Teilbereiche und ihrer Diskurse mussten von anderen Sprecherpositionen aus zu anderen Synthesen als bisher zusammengeführt werden.

Genau diese Lücke füllte zwischen 1959 und 1968 für eine Spanne von knapp zehn Jahren Walter Höllerer aus, und zwar mit seinem Projekt der Kombination und teilweisen Integration öffentlichkeitsrelevanter literarisch-medialer Arbeitsfelder unter weitgehendem Ausschluss von >großer Politik, aber unter Einschluss der Literatur- und im Weiteren auch Kulturpolitik Berlins. ${ }^{7}$ Dabei positionierte sich Höl-

6 Siehe dazu Link, Jürgen: >Diskurs $\prec$ und/oder $>$ Ideologie $<$ ? In: kultuRRevolution 4 (1983), S. 46-68; ders.: Warum Foucault aufhörte, Symbole zu analysieren: Mutmaßungen über >Ideologie` und >Interdiskurs`. In: Dane, Gesa u.a. (Hg.): Anschlüsse. Versuche nach Michel Foucault. Tübingen 1985, S. 105-114; ders.: Das Gespenst der Ideologie. In: Baßler, Moritz / Gruber, Bettina / Wagner-Egelhaaf, Martina (Hg.): Gespenster. Erscheinungen, Medien, Theorien. Würzburg 2005, S. 335-347.

7 Hans Werner Richter (Das Lachen aus der Oberpfalz. Walter Höllerer. In: Ders.: Im Etablissement der Schmetterlinge. Einundzwanzig Portraits aus der Gruppe 47. München 1986, S. 149-159) hat Höllerer in ganz ähnlicher Perspektive als jemanden charakterisiert, der auf lokaler Ebene einen $» B e-$ hörden Sex-Appeal« habe (S. 156), der aber innerhalb der Gruppe 47 »eine Art Fraktionsbildung« gegen die »politisch engagierte Literatur der ersten Jahre, die es immer noch gab« betrieben habe (S. 158). Vgl. auch Neumann 1966, S. 37: »[...] Höllerer ist etwa so politisch wie Löschpapier oder Watte in einem Ärzteschrank [...].« - Vgl. zu Berlin als Zentrum 
lerer innerhalb >seiner Integrationskonstellation sehr geschickt, indem er über seine Arbeitsfelder hinweg rotierte und dadurch weder für ein einziges vereinnahmt werden konnte, noch Gefahr lief, als übermächtiger Integrator zu erscheinen, der alle Felder zu besetzen in der Lage gewesen wäre, und dies auch noch als Experte. Nicht nur die Kombination von in der Regel eher arbeitsteilig verfolgten Bereichen des Literaturbetriebs machte demnach die Spezifik Höllerers als Intellektueller im Literaturbetrieb seiner Zeit aus, sondern ein Rotieren innerhalb des gesamten Spektrums der von ihm verfolgten Teiltätigkeiten, wobei als weiteres konstitutives Element tendenziell auch noch die gegenläufige Besetzung der einzelnen Arbeitsbereiche hinzukam, also die Strategie, auf einem Platz - wenn irgend möglich - eine nicht genuin damit verbundene und gerade nicht erwartete Position einzunehmen. So trat er bei der Gruppe 47 unter lauter Schriftstellern zunächst über längere Zeit hinweg als Kritiker, Organisator, aber auch »Einkäufer« für die Akzente auf, ${ }^{8}$ weniger jedoch als Dichter, was zu einem hohen Grad an Ambivalenz zwischen Beobachter- und Mitgliedsstatus führte. Umgekehrt betätigte sich der Lyriker Höllerer als Herausgeber, der Wissenschaftler an der Technischen Universität Berlin als Literat, der literarische Autor in Rundfunk und Fernsehen als Wissenschaftler, der Germanist als Komparatist. ${ }^{9}$ Man könnte das als eine Strategie des Sich-Dislozierens bezeichnen, die allerdings so angelegt war, dass das Sich-Dislozierende-Rotieren zu einem Effekt von Integration führte, und zwar zu umso intensiverer, je schneller und öfter die Wechsel zwischen den einzelnen Arbeitsbereichen erfolgten.

Solche mehrfachen Positionswechsel hat Claude Lévi-Strauss als eine gegenüber der Figur des gesellschaftlich relevante Gegensätze in sich vereinenden Trickster als zwar schwächere, jedoch ebenfalls durchaus wirkungsvolle Form des imaginären Ausgleichs zwischen

amerikanischer kulturpolitischer Interventionen auch den Beitrag von Michael Peter Hehl in diesem Band.

8 Richter 1986, S. 151: »Er [Höllerer, R.P.] betätigte sich auch als Einkäufer, denn er gab die Zeitschrift `Akzente〈 heraus und ließ drucken, was seinem literarischen Urteil standhielt.«

9 Vgl. auch den Beitrag von Sven Hanuschek in diesem Band, der von `Erwartungsirritationen spricht. 
eigentlich antagonistischen Positionen beschrieben. ${ }^{10}$ Im Falle Höllerers scheint es dabei gerade die Schriftsteller-, d.h. Künstlerseite, gewesen zu sein, die ihn für die Tätigkeit als Literaturvermittler prädestinierte, denn sie stellte sicher, dass er trotz aller wissenschaftlichen Fortschrittlichkeit und insbesondere Offenheit für Medial-Technisches stets auch als jemand angesehen werden konnte, der die ältere, aus dem 19. Jahrhundert resultierende Bestimmung des Geisteswissenschaftlers als Künstler weiter tradierte. Dieses Konzept war bis zum Zweiten Weltkrieg nicht untypisch, man denke etwa an den GeorgeKreis. ${ }^{11}$ Während Künstler-Wissenschaftler vom Typus eines Friedrich Gundolf »den Antagonismus zwischen Kunst und Wissenschaft also durch seine Wiederholung innerhalb der Wissenschaft selbst ${ }^{12}$ reproduzierten, konnte Höllerer Kunst und Wissenschaft durch seinen wissenschaftlichen Heimatort an einer Technischen Universität und durch die Semiologie als ein vom Anspruch her dezidiert szientistisches Theorieinstrument sowie durch sein Agieren auf institutionell und auch räumlich ganz verschiedenen Bühnen zugleich voneinander trennen und wirkungsvoll aufeinander beziehen.

So betrachtet hat Höllerer die Vorkriegskonstellation des Bedingungsdreiecks von Literatur (im Weiteren Kunst überhaupt), Literaturwissenschaft und >harter< Natur- bzw. Ingenieurwissenschaften dadurch modernisiert, dass er das >alte`, synchron integrierende Denkmodell von >Wissenschaft als Kunst vs. unkünstlerische harte Wissenschaften $<$ in das neue Modell eines auch technisch-mediale Aspekte einbeziehenden rotierenden Positionswechsels im Nacheinander überführt. Dafür gab sein eigenes Leben und Agieren zugleich die sinnfällige Anschauungsform ab. Auf diese Weise war auf keinem einzelnen seiner Arbeitsfelder die Reputation durch das Image eines der jeweils anderen gefährdet, Dichter und Wissenschaftler, Literat und Literaturvermittler zu sein, waren keine Gegensätze mehr. Das war

10 Vgl. dazu Parr, Rolf: »Zwei Seelen wohnen, ach! in meiner Brust.« Strukturen und Funktionen der Mythisierung Bismarcks (1860-1918). München 1992, bes. S. $24 f$.

11 Ich folge hier einer Überlegung, die Ulrike Pisiotis in ihrer Dissertation Rivalität und Bündnis. Zum Verhältnis von Wissenschaft und Literatur der Moderne, Dresden 2012 (= Kulturstudien, Bd. 7) in anderem Zusammenhang entwickelt hat.

12 Ebd., S. 93. 
gegenüber der seit Beginn der klassischen Moderne geführten Diskussion um das Verhältnis von Kunst und Wissenschaft eine neue, kreative, wenn auch eher auf vermeidende Ausgrenzung der drohenden Konflikte setzende Innovation. Erleichtert wurde sie durch die ab Ende der 1950er Jahre für einige Zeit vermehrt auch für Literatur zur Verfügung stehenden Sendeplätze in Rundfunk und Fernsehen. Von daher scheint es mir nicht ganz richtig bzw. Teil einer gewissen HöllererMythisierung zu sein, ihn wie beispielsweise Hermann Schlösser ${ }^{13}$ als >dichtenden Germanistikprofessor` zu bezeichnen (mit der dahinter stehenden Idee, dass die eine Seite von der anderen nicht zu trennen ist) oder wie Norbert Miller zu konstatieren, dass Höllerer »den Dichter, den Essayisten, den Wissenschaftler und den um Einsicht werbenden Publizisten nie getrennt « habe. ${ }^{14}$

Demgegenüber soll im Folgenden an einigen Beispielfeldern aus Höllerers Tätigkeitsspektrum gezeigt werden, dass er mehr rotierte als intensiv-semantisch synthetisierte, dass er entweder Germanistikprofessor oder Dichter war, entweder Dichter oder Literaturvermittler, was partielle kleinere Integrationen auf einer subdominanten Ebene jedoch überhaupt nicht ausschloss. Solche partiellen Kopplungen lassen sich teilweise bis ins Detail hinein verfolgen. Sie stellen für Höllerer die Bedingung der Möglichkeit dar, in den 1950er und 1960er Jahren als eine Art >Spinne im Netz des Literaturbetriebs`zu erscheinen, und zwar ohne die zu diesem Zeitpunkt als Position noch unmögliche >große Synthese < je wirklich leisten zu müssen (und dies wohl auch weder gewollt, noch taktisch für besonders klug erachtet zu haben).

Das erste dieser partiellen, manchmal auch nur punktuellen Kopplungs- und/oder Rotationsprojekte ist das von Dichter und Kritiker. Seinen Kern findet man in der Struktur der 1954 gegründeten und für

13 Schlösser, Hermann: Literaturgeschichte und Theorie in der Literatur. In: Hansers Sozialgeschichte der deutschen Literatur vom 16. Jahrhundert bis zur Gegenwart. Hg. von Rolf Grimminger. Bd. 12: Gegenwartsliteratur seit 1968. Hg. von Klaus Briegleb und Sigrid Weigel. München 1992, S. 385403, hier S. 395.

14 Miller, Norbert: Vorwort. In: Ders. / Klotz, Volker / Krüger, Michael (Hg.): Bausteine zu einer Poetik der Moderne. Festschrift für Walter Höllerer. München 1987, S. 7-10, hier 8. - Völlig richtig dagegen dann die Fortsetzung des Gedankens: »Nur im Zusammenspiel der Möglichkeiten sah er die Chance, für die Zukunft der Literatur zu wirken« (ebd.). 
lange Zeit zusammen mit Hans Bender herausgegebenen »Akzente. Zeitschrift für Dichtung «, ${ }^{15}$ die »von vornherein auf« ein »Ineinander von Dichtung und poetologischer Einsicht« sowie Kritik (letztere im Sinne des weiten angloamerikanischen Begriffs >criticism` zu verstehen) »ausgerichtet« war. $^{16}$ In der Gruppe 47, also eigentlich auf dem engeren Feld der Literatur, kam zu diesem Kern das Element der rotierenden Besetzung ganz verschiedener Felder hinzu, fungierte Höllerer innerhalb der Gruppe doch zunächst als Literaturkritiker, dann als Organisator und Herausgeber der Akzente (die den anderen Mitgliedern einen Publikationsort boten) und erst später als Dichter unter Dichtern.

Eng mit diesem ersten Teilprojekt zusammen hängt - zweitens die Kombination von Dichter und Wissenschaftler, die hier nur symptomatisch belegt sein soll. Von Pierre Chevalier zu einem Vortrag eingeladen, antwortete Höllerer auf eine Weise - darauf haben Roland Berbig und Alexander Krüger hingewiesen -, die den Literaturwissenschaftler und den Autor zugleich ins Spiel brachte, und zwar auch hier wieder nicht wirklich integral, sondern in Form des Positionswechsels im Nacheinander. In Hölleres Schreiben heißt es:

Als Thema würde ich vorschlagen: »Deutsche Lyrik im 20. Jahrhundert und einige Verbindungslinien zur französischen und englischen Lyrik«. Ich würde in diesem Rahmen auf Ihre beiden Leitbegriffe »engagement« und »révolte« eingehen und, wenn Sie es wünschen, auch aus eigenen Texten lesen. Falls Ihnen das lieber ist, kann ich neben dem Vortrag am nächsten Tag eine Lesung aus eigenen Texten abhalten. Man kann aber auch beides kombinieren. ${ }^{17}$

Die prinzipiell auch denkbare Alternative snur Wissenschaft ohne Lesung « wird damit gar nicht mehr ernsthaft erwogen, sondern nur die Kombination beider. Auch hier haben wir es wieder mit einer der partiellen Integrationen im Gesamtfeld des Tätigkeitsspektrums von Höl-

15 Akzente. Zeitschrift für Dichtung. Hg. von Walter Höllerer und Hans Bender, Jg. 1 (1954).

16 Miller 1987, S. 9.

17 Höllerer, Walter an Pierre Chevalier: Brief v. 3.3.1959. Zit. n.: Berbig, Roland / Krüger, Alexander: Ein Novum unter der Ägis eines Lehrstuhlinhabers. Walter Höllerer im Jahr 1959. In: Berliner Hefte 8 (2008), S. 8999, hier S. 96. 
lerer in Form des Rotierens zu tun, hier praktiziert als einfacher Positionstausch.

Was den Wissenschaftler Höllerer angeht, ist - drittens - die Verknüpfung von Literaturwissenschaft, Linguistik und Technik relevant, die ein weiteres Projekt partieller Integration bildet, das auf dem Feld der Semiologie als gemeinsamer Bezugstheorie realisiert werden sollte, um die »Sprache im technischen Zeitalter« an der Technischen Hochschule Berlin adäquat zu erforschen. In Höllerers »Memorandum zur Gründung eines Instituts `Sprache im technischen Zeitalter«« vom 8. Dezember 1959 heißt es:

Ein weiterer Bereich der wissenschaftlich-theoretischen Untersuchungen dieses Instituts wird sein, die Brücke von der Sprache zur modernen Literatur zu schlagen: Wie hat sich die moderne Literatur dem technischen Sprechzustand angepasst, welche Gegenkräfte hat sie dagegen entwickelt, wie sind die $\mathrm{Zu}$ sammenhänge zwischen »Sprache im technischen Zeitalter « und der modernen Lyrik, der modernen Prosa, der modernen Dramatik und der modernen Kritik. Eine Abteilung des Instituts wird sich vor allem mit der literarischen Kritik als Sprachkritik befassen, auch mit der kritischen Auseinandersetzung mit den spezialisierten Fachsprachen, der Diktion von Fachaufsätzen, nicht zuletzt mit der politischen Rhetorik im technischen Zeitalter und der Sprache des Kulturbetriebs. Von einer solchen Aktivität kann ein neuer Impuls für eine gemeinsame kritisch wissenschaftliche Grundlegung der speziellen Wissenschaften erwartet werden. ${ }^{18}$

Dass es aber auch hier nicht um intensive Synthese, sondern wiederum um ein Rotieren zwischen den Perspektiven von Linguistik und Literatur sowie zugleich von Kunst (Dichtung) und Technik geht, macht die Ergänzung von »Sprache im technischen Zeitalter« durch »Literatur im technischen Zeitalter« deutlich. Gedacht war Sprache im technischen Zeitalter, wie Höllerer in seiner Dankesrede zur Verleihung der Ehrenmitgliedschaft der Technischen Universität Berlin ausführte, als Komplementärstück zu Akzente. Höllerer in seiner »Erwiderung«: »Sie sollte durch Essay den Disput fördern. Wie verwiesen in ihr auf die gesellschaftlichen Veränderungen, die das elektronische Zeitalter auf

18 Höllerer, Walter: Memorandum zur Gründung eines Instituts »Sprache im technischen Zeitalter« vom 8.12.1959. Abgedruckt in: Berliner Hefte 8 (2008), S. 103-109, hier S. 107. 
allen Gebieten des täglichen Lebens [...] mit sich brachte. « ${ }^{19}$ Diese Kopplung von Literatur, Literaturwissenschaft und Linguistik sowie Technik und Technikbewusstsein musste konsequenterweise auch die Medien einbinden. Hier ist - viertens - insbesondere die Kopplung von Lyrik mit Medien wie Fernsehen, Radio und Schallplatte zu nennen. Zugleich bedeutete dies eine Verbindung von »Hochkultur mit einem Showcharakter «, ${ }^{20}$ wie ihn die moderierte Lesung unweigerlich mit sich brachte. Die Verknüpfung gelang Höllerer für eine gewisse Zeit produktiv und ohne die von Arnold Gehlen schon früh diagnostizierte Gefahr, als literarisch-wissenschaftlicher Intellektueller durch die modernen Medien zum »Entertainer des Publikums« gemacht zu werden, also seine als »authentisch« wahrnehmbare »Individualität« zu verlieren. ${ }^{21}$

Am deutlichsten wird das Modell rotierender Integration vielleicht - fünftens - an Höllerers Herausgeber- und das heißt vor allem Anthologistentätigkeit, die es ihm erlaubte, souverän zwischen Wissenschaft, Literatur und Essayismus hin und her zu wechseln, also Schnittstellen herzustellen, um sie gleich wieder zu unterlaufen. Symptomatisch sind hier bereits die Titel der von Höllerer herausgegebenen Anthologien. Im Falle von »Ein Gedicht und sein Autor. Lyrik und Essay. Hg. und mit Einleitungen versehen von Walter Höllerer« ist es der Plural bei »Einleitungen ${ }^{22}$ der nicht nur untypisch für die Gattung ist, sondern vor allem den kommentierenden und interpretierenden Literaturwissenschaftler in einem Feld stark macht, das eigentlich als eines von Autoren untereinander (ja sogar in der emphatischen Variante der Lyriker untereinander) gilt, und bei dem sich der herausgebende Wissenschaftler auf einen einleitenden Part zu beschränken hat. Oder als zweites Beispiel »Berlin: Literarisches Transit. Lyrikbuch der Jahr-

19 Höllerer, Walter: Erwiderung. In: Walter Höllerer zu Ehren. Festveranstaltung anlässlich der Verleihung der akademischen Würde Ehrenmitglied der Technischen Universität Berlin. Berlin, 12. Februar 1993 (= TUB DOKUMENTATION. Kongresse und Tagungen, H. 62), S. 33-42, hier S. 41.

20 Böttiger, Helmut: Elefantenrunden. Walter Höllerer und die Erfindung des Literaturbetriebs. Berlin 2005, S. 8.

21 Jäger 2000, S. 24.

22 Höllerer, Walter (Hg.): Ein Gedicht und sein Autor. Lyrik und Essay. Berlin 1967. 
hundertmitte. Hg. mit Randnotizen von Walter Höllerer «, ${ }^{23}$ in denen er die einzelnen Gedichte assoziativ fortführt. Hier haben wir das gleiche Muster.

Kritisch ließe sich insgesamt einwenden, dass man das auch als eine Form von Selbstmythisierung verstehen kann, deren Deckung nicht immer gegeben ist. So erscheinen die im Plural angekündigten Texte hinsichtlich ihres rein literaturwissenschaftlichen Ertrags bisweilen doch eher zurückhaltend. ${ }^{24}$ Doch eine Dominanz des Literaturwissenschaftlers Höllerer ist in diesem Falle ebenso wenig intendiert wie beim Lyrikbuch »Transit«. Hier ist Höllerer Herausgeber, Vorwortschreiber, Beiträger (einer der mit 11 Gedichten am häufigsten vertreten ist) und auch noch Kommentator auf der Schwelle zwischen Literaturwissenschaft, Merksatz, ästhetischem Glaubensbekenntnis, Aphorismus und Essay, wobei die Favorisierung des Essays im interdiskursiven Charakter dieser Textsorte liegt. Das Projekt rotierender Integration und - sechstens - der Rückgriff auf die Textsorte Essay stützen sich also wechselseitig. $\mathrm{Zu}$ fragen ist, warum diese Kombination auf der Hand lag. Darauf soll in einem kleinen Exkurs zum Essayismus als synthetisierendem Schreibverfahren eingegangen werden. ${ }^{25}$

Wenn die Besonderheit von Literatur in ihrer je spezifisch realisierten Form der Zusammenführung mehrerer Spezialbereiche und ihrer Diskurse besteht, dann ist auch der Essay als literarische Form ein

23 Höllerer, Walter (Hg.): Transit. Lyrikbuch der Jahrhundertmitte. Frankfurt a.M. 1956

$24 \mathrm{Zu}$ einem ähnlichen Befund kommt auch Michael Kämper-van den Boogaart (»Und einmal muß es gesagt werden...« Der Autor und Germanist Walter Höllerer im Dienste der Gruppe 47. Ein Vorfall aus dem Jahr 1966. In: Zeitschrift für Germanistik 17, 2007, H. 1, S. 108-127, hier 124f.) für Höllerers Aufsatz Zur Sprache im technischen Zeitalter, veröffentlicht in dem unter dem Pseudonym Friedrich Handt von Höllerer selbst herausgegeben LCB-Band Deutsch - gefrorene Sprache in einem gefrorenen Land. Polemiken und Aufsätze (Berlin 1964), S. 186-200, in dem zwar eine Art vor-diskurstheoretische Metapherntheorie entfaltet, nicht aber auch nur der Ansatz zum Versuch einer Metaphern-Definition unternommen wird.

25 Vgl. dazu ausführlich Parr, Rolf: `Sowohl als auch` und `weder noch`. Zum interdiskursiven Status des Essays. In: Braungart, Wolfgang / Kauffmann, Kai (Hg.): Essayismus um 1900. Heidelberg 2006, S. 1-14. 
eminent interdiskursives, einzelne Spezial- und Interdiskurse auf vielfältige Weise verbindendes Genre (und der Essayismus ein ebensolches Schreibverfahren). Denn essayistisches Schreiben bietet eine Möglichkeit, der »Isolierung der Diskurse durch die rasante Spezialisierung in allen Bereichen der Wissenschaft und der Gesellschaft« entgegenzuwirken, »es fordert auf zu grenzüberschreitender Kommunikation $\ll{ }^{26}$ Bezieht man nun die Frage des Changierens essayistischer Schreibweisen zwischen den beiden Polen >Wissenschaft und >Literatur auf die Unterscheidung von Spezialdiskursen und literarischem Interdiskurs, dann wird man sagen müssen, dass der Essay zwar ebenso wie Literatur überhaupt interdiskursiv dominiert ist, aber doch zugleich auch noch einen nicht unerheblichen Rest an spezialdiskursiver Ausrichtung aufweist. Das bedeutet: Je stärker man den Essay an den Pol > Wissenschaft $<$ bindet und je weniger an den der >Literatur $<$, umso mehr ist er auf Denotation und Eindeutigkeit und umso weniger auf Konnotationsreichtum, semantische Vieldeutigkeiten und Mehrfachlesarten hin angelegt. Genau umgekehrt sieht es aus, wenn man den literarischen Charakter des Essays besonders stark betont. Dann teilt der Essay seine hochgradige Interdiskursivität mit derjenigen von Literatur überhaupt, aber immer noch mit der differenzierenden Spezifik, dass es für den Essay die Kombinationsvorgabe gibt, genuin geisteswissenschaftliche Themen auf in der Regel ungewöhnliche Weise mit tendenziell politischen und/oder wissenschaftlichen Gegenständen und Redeformen zu koppeln und dabei Brüche, Sprünge, ja sogar Friktionen nicht nur in Kauf zu nehmen, sondern geradezu zu suchen und zu forcieren (auch solche zwischen konnotativen und denotativen Textelementen). Das ist sowohl in der synchronen Kombination der Themen der verschiedenen Praxisbereiche und der mit ihnen verknüpften je spezifischen Redeformen, als auch in der Diachronie der Durchführung der Fall.

Zusammenfassend lässt sich demnach sagen: Essays bzw. essayistische Schreibweisen zeichnen sich dadurch aus, dass sie zugleich sowohl ungebrochen spezialdiskursives Material als auch hochgradig interdiskursives, insbesondere literarisches Diskursmaterial verarbeiten. Dadurch treffen auf Eindeutigkeit zielende wissenschaftliche

26 Pfammatter, René: Essay - Anspruch und Möglichkeit. Plädoyer für die Erkenntniskraft einer unwissenschaftlichen Darstellungsform. Hamburg 2002, S. 16. 
Passagen auf vieldeutige und mehrfach anschließbare literarische Elemente, Spezialdiskurse auf Interdiskurse. Beide werden nicht immer und nicht immer vollständig miteinander vermittelt, sondern nur teilweise, sodass der Essay manche Übergänge auch in Form von gewollten Brüchen realisiert, etwa wenn mehrmals mit neuen Denkbewegungen angesetzt wird, sodass die Brückenschläge immer nur für den $\mathrm{Au}$ genblick Gültigkeit haben und meist sofort wieder durch anders und neu zusammengefügte imaginäre Ganzheiten abgelöst werden. Analytisch lassen sich diese Bestimmungen des Essayistischen an zahlreichen Texten Höllerers schnell belegen. So weit zum essayistischen Schreiben als ein weiteres in der Reihe der kleinen Integrationsprojekte Walter Höllerers.

Biografisch betrachtet kommt zu den bisherigen Projekten - siebtens - noch das der wechselnden Wohnsitze bzw. Arbeitsorte hinzu. So schreibt Herbert G. Göpfert in der Festschrift »Bausteine zu einer Poetik der Moderne«, dass es Höllerer schon zum Zeitpunkt der Gründung der Akzente wichtig gewesen sei

an mehreren Orten Domizile zu haben und je nach Belieben oder nach Arbeitsvorhaben hier oder dort zu leben: in Erlangen, wohin er noch von den letzten Studiensemestern her Beziehungen hatte, in Frankfurt, wo er an der Universität tätig war, und in Heidelberg, wohin er sich gern zu seinen eigenen Angelegenheiten zurückzog. ${ }^{27}$

An dem im Höllerer-Archiv in Sulzbach-Rosenberg (das Göpferts Liste noch hinzuzufügen wäre) vorhandenen Material wäre gelegentlich einmal genauer zu überprüfen, ob und wie einzelne Aufenthaltsorte mit je speziellen Arbeitsfeldern korrelierten.

Achtens schließlich dienten Höllerer auch Pseudonyme dazu, das Rotieren und zugleich das Prinzip der gegenläufigen Besetzung von Arbeitsfeldern in noch einmal zugespitzter Form zu praktizieren. Den LCB-Band Deutsch - gefrorene Sprache in einem gefrorenen Land. Polemiken und Aufsätze gab Höllerer - darauf hat Michael Kämpervan den Boogaart hingewiesen ${ }^{28}$ - unter dem Pseudonym »Friedrich

27 Göpfert, Herbert G.: »Akzente« - vorgeschichtlich. In: Miller / Klotz / Krüger 1987, S. 99-105, hier 102.

28 Vgl. Kämper-van den Boogaart 2007, S. 122-124. 
Handt « heraus, ${ }^{29}$ veröffentlichte darin unter diesem Pseudonym einen Beitrag, ${ }^{30}$ zugleich aber auch einen weiteren mit dem Titel Zur Sprache im technischen Zeitalter als Walter Höllerer, während das Nachwort dann wieder mit »Friedrich Handt« unterzeichnet ist. ${ }^{31}$ Damit ist das Prinzip des Sich-Dislozierens maximal ausgeschöpft, der Integrationseffekt wird aber zugleich geringer, kann nur noch erzielt werden, wenn man das Pseudonym aufzulösen weiß.

Wie wichtig die Mischung aus dem Spektrum der acht hier aufgezeigten kleinen Kopplungen sowie der Rotation und gegenläufigen Positionsbesetzung für Höllerer war, zeigt sich in solchen Momenten, in denen er sich nicht mehr darauf zurückziehen, nicht mehr gleichsam spielerisch über seine verschiedenen `Identitäten` verfügen konnte. Eine solche Situation stellte seine erste Lesung aus dem Roman »Die Elephantenuhr $\aleph^{32}$ beim Treffen der Gruppe 47 in Elmenau 1959 dar, von der Hans Werner Richter rückblickend berichtet:

Ich erinnere mich noch sehr genau an diesen Augenblick. Ich rief ihn zur gegebenen Zeit auf, und er strebte sogleich auf den Stuhl zu, der neben mir stand, mit seinem Romanmanuskript in der Hand. Er war sehr aufgeregt. Ich hatte ihn noch nie und habe ihn auch später nie wieder so gesehen. Er schien seine Selbstbeherrschung verloren zu haben, ja, es ist mir damals vorgekommen, als sei er der Auflösung nahe. Sein Gesicht war fahl und seine Nase, seine bemerkenswerte und - so meine ich - bedeutende Nase war fast grün. Natürlich war es schwerer für ihn, als für andere. Er hatte sich seit Jahren an der Kritik beteiligt und sein Auftritt als Romancier würde sicher schärfer und genauer beurteilt

29 Handt [d.i. Höllerer] 1964. - Höllerer verwendet dieses Pseudonym gelegentlich auch in Sprache im technischen Zeitalter.

30 Handt, Friedrich: Das hohle Wunder. Bericht über drei Aufsätze von George Steiner John, McCormick und Hans Habe. In: Ders. 1964, S. 9-26.

31 Höllerer 1964; Handt, Friedrich: Nachwort: In: Ders. 1964, S. 201. - Auch in Sprache im technischen Zeitalter taucht das Pseudonym da auf, wo ein Heft ganz pragmatisch mit Beiträgen Höllerers gefüllt werden musste, aber nicht zu viele Artikel mit seinem Namen erscheinen sollten (ich danke Thomas Geiger, dem heutigen Redakteur der Zeitschrift, für diesen Hinweis).

32 Höllerer, Walter: Die Elephantenuhr. Roman. Frankfurt am Main 1973. 
werden als die Arbeit irgendeines Unbekannten. [...] Was es auch immer war, für ihn war in diesem Augenblick der >elektrische Stuhl eine Art Prüfstand. ${ }^{33}$

Das ritualisierte Setting des sogenannten >elektrischen Stuhls`, auf dem jeder Lesende Platz zu nehmen hatte, machten das von Höllerer sonst auch innerhalb der Gruppe 47 praktizierte rotierende Dislozieren unmöglich, was zu jenem vorübergehenden Souveränitätsverlust führte, den Richter sehr genau beschrieben hat.

Insgesamt wird man sagen können, dass Höllerer keineswegs derjenige war, der alles mit allem im Literaturbetrieb vernetzt hat, wohl aber jemand, der sich selbst und sein Tätigkeitsspektrum als ein maximal breit angelegtes Integrationsprojekt inszeniert und darin partielle kleinere Kopplungsprojekte realisiert hat. Das zeigt sich vor allem im Rotieren quer durch die einzelnen Arbeitsfelder und in der Selektivität bzw. Partialität der Kopplungsprojekte, von denen manche auch auf der Ebene programmatischer Absichtserklärungen stehen geblieben sind. ${ }^{34}$ Tendenziell kann man beispielsweise Höllerers Orientierung an der Semiologie als Element eines integrierenden Überbaus ansehen, der aber nicht bis in seine wissenschaftlichen Texte durchschlägt, die doch eher einer traditionellen Hermeneutik auf Basis genauester Textkenntnis folgen. Von daher besteht eine gar nicht gering zu erachtende Leistung Höllerers darin, >alt` und >neu zu Beginn der 1960er Jahre miteinander konfrontiert und so das Terrain für die linguistische Wende der Literaturwissenschaft in den 1970er Jahren vorbereitet zu haben.

Insofern sich Höllerers Rotieren über verschiedene Arbeitsfelder, das verschiedene gesellschaftliche Teilbereiche und ihre je speziellen Diskurse integrierend ins Spiel bringt (und zwar ohne ex post >große Synthesen $<$ zu propagieren) mit Claude Lévi-Strauss als eine Form der

33 Richter 1986, S. 151.

34 Ein Symptom dafür, dass Höllerer eben kein `Knotenpunktく im Sinne des New Historicism war, liegt darin, dass er in den am New Historicism orientierten Literaturgeschichten nicht vorkommt. Vgl. Wellbery, David E. u.a. (Hg.): Eine neue Geschichte der deutschen Literatur. Berlin 2007. 
Mythisierung verstehen lässt, also als Kombination von eigentlich nicht zugleich vorkommenden antagonistischen Elementen in einer Figur, die dadurch zwangsläufig gegenüber anderen überhöht wird, ist es auch hier nicht ganz unpassend von Selbstmythisierung zu sprechen.. Dieser Befund muss bei Höllerer jedoch gegenüber echten Trickstern, die Gegensätze in sich vereinen, auf das Modell der schwächeren Integration des rotierenden Positionstausches beschränkt bleiben. Eine demgegenüber sehr viel stärkere Synthese und damit auch sehr viel stärkere Form der Mythisierung stiftet erst die HöllererRezeption, indem sie das Rotieren im Nacheinander in das Bild eines personalisierten mythischen Tricksters Walter Höllerer verwandelt, bei dem alle Facetten stets zugleich anzutreffen sind. ${ }^{35}$

Zusammenfassend kann man sagen, dass Höllerer keine Integration im Sinne semantisch-intensiver Zusammenführung von arbeitsteiligen gesellschaftlichen Teilbereichen betrieben hat, sondern als eine Art Manager von der >Homebase verständnisses aus rotierte und dabei Inhalte bisweilen durch Funktionen ersetzte (mit Ausnahme des Gebiets der Dichtung). Integration der Felder gelang nicht in den Texten, weder den literarischen, noch den wissenschaftlichen, sondern in der Praxis des Rotierens über viele Funktionspositionen hinweg: viel Verschiedenes tun, es aber nie gleichzeitig tun, sodass Integration nur im zeitlichen Nacheinander entsteht. Dem entspricht recht genau die Bewegungsmetaphorik der »Transit«-Anthologie.

\section{IV}

Dieses Spektrum der Projekte partieller Kopplungen, das man auf die Formel >Autor plus Wissenschaftler plus Medien minus Politik gleich Intellektueller im Literaturbetrieb ‘ bringen könnte (und damit auch die Integration des Gesamtspektrums der von Höllerer besetzten Felder im Literatur- und Wissenschaftsbetrieb), funktionierte so lange reibungslos, bis es durch die Studentenbewegung vehement infrage gestellt wurde. Denn eine der Voraussetzungen dafür, dass Höllerers Rotationsprojekt in den späten 1950er und frühen 1960er Jahren überhaupt funktionierte, war der weitgehende Ausschluss von >großer Politik, was Hans Werner Richter geradezu als Wesenszug Höllerers beschrie-

35 Siehe als Beispiel das Zitat zu Anm. 12. 
ben hat, da er sich auch in der Gruppe 47 nie an politischen Diskussionen oder Protesten beteiligt habe. ${ }^{36}$ Politik, ja Weltpolitik, forderte die Studentenbewegung aber gerade ein und machte sie zum Bewertungsmaßstab gerade auch von Literatur. Von daher verwundert es nicht, dass Höllerer 1967 bei der Tagung der Gruppe 47 mit lauten »Dichter, Dichter«-Rufen ausgebuht wurde. ${ }^{37}$

Auch nach Höllerer besteht das Feld der für den Literaturbetrieb relevanten Teilbereiche strukturell nahezu identisch weiter, wird aber von den Akteuren nicht mehr in toto zu besetzen gesucht. Vielmehr bilden sich speziellere Kopplungen mit höherem Professionalitätsgrad für eingeschränktere Teilbereiche des Literaturbetriebs heraus: Marcel Reich-Ranicki etwa, um ein bekanntes Beispiel zu nehmen, koppelt seine Rezensententätigkeit in der Presse mit der im Fernsehen und schafft es ebenso wie Höllerer auf ganz andere Art Unverwechselbarkeit gerade als literarischer Entertainer des Publikums zu entwickeln, sich gleichsam selbst zum Markenprodukt zu machen. Das Element des praktizierenden Literaten bleibt dabei anders als bei Höllerer weitgehend außen vor. Man könnte weitere Fälle durchgehen, von Hellmuth Karasek über Sigrid Löffler bis hin zu Elke Heidenreich. Wie diese Reihung zeigt, sind die >zünftigen ২ Literaturwissenschaftler in den Medien eher im Rückzug begriffen. Dafür sind die Schriftsteller selbst inzwischen fast alle gelernte Germanisten, man denke nur an F.C. Delius, den Höllerer promoviert hat. Zudem nutzen Schriftsteller die Medien zunehmend offensiver für sich selbst, wofür Martin Walser, der im Februar 1956 in einem Brief an Höllerer das Fernsehen noch als Wurzel allen Übels anprangerte, ${ }^{38}$ bis heute ein durchaus hin-

36 Richter 1986, S. 157: „Walter Höllerer unterschrieb keinen dieser Proteste. [...] Ja, er hätte nur seinen eigenen Protest unterschrieben [...]. [...] seinerzeit $[\ldots]$ hielt ich es für das Zeichen eines völlig unpolitischen Menschen.« - Wollte man es genauer machen, so wäre für verschiedene Zeitpunkte zu unterscheiden, welche Mitglieder der Gruppe sich auf welche Weise in und außerhalb des Zirkels politisch äußerten, und wie dies von außen wahrgenommen wurde.

37 Siehe dazu Briegleb, Klaus: 1968. Literatur in der antiautoritären Bewegung. Frankfurt am Main 1993 (es 1669), S. 124.

38 Vgl. Walser, Martin an Walter Höllerer: Brief v. 6.2.1956. In: Literaturarchiv Sulzbach-Rosenberg (LSR), Vorlass Walter Höllerer, Signatur: 02WH/142/10. Michael Peter Hehl danke ich für den freundlichen Hinweis 
terfragbares, aber doch in seiner Art auch eindrucksvolles Beispiel darstellt. Hinzu kommt, dass sich die Rezipienten von Literatur bereits in den 1960er Jahren in verschiedenste Milieus ausdifferenziert hatten, ${ }^{39}$ was so breit angelegten Publikumsprojekten wie dem des literarisch-wissenschaftlichen Intellektuellen Höllerer abträglich sein musste, denn die relative Geschlossenheit der (literarischen) Milieus bildete eine der Voraussetzungen für den Erfolg des von ihm praktizierten Modells. Dislozierende Rotation bei zugleich hochgradig ausdifferenzierten Rezipientenmilieus wäre heute kaum mehr erfolgreich zu betreiben. Dieser Befund gilt - last but not least - ähnlich auch für die Wissenschaft. Denn die Realisierung des von Höllerer intendierten semiologischen Programms hat an der Technischen Universität Berlin bereits die ihm nachfolgende bzw. sich mit ihm überlappende Wissenschaftlergeneration nur noch spezialisiert übernommen: Friedrich Knilli in Form der Filmsemiotik, Roland Posner für die allgemeine Semiotik, was in beiden Fällen aber unweigerlich mit zunehmender Spezialisierung der Theoriebildung einherging.

auf diesen Brief. - Vgl. auch Parr, Rolf: Paradoxe Medienästhetik. Martin Walsers >Realismus $\measuredangle$ und sein literarischer Umgang mit Film und Fernsehen. In: Geisenhanslüke, Achim / Mein, Georg / Schössler, Franziska (Hg.): Das Subjekt des Diskurses. Festschrift für Klaus-Michael Bogdal zum 60. Geburtstag. Heidelberg 2008, S. 303-320.

39 Vgl. dazu u.a. Bogdal, Klaus-Michael: Klimawechsel. Eine kleine Meteorologie der Gegenwartsliteratur. In: Erb, Andreas (Hg.) unter Mitarbeit von H. Krauss u. J. Vogt: Baustelle Gegenwartsliteratur. Die neunziger Jahre. Opladen / Wiesbaden 1998, S. 9-31. 


\section{QUELLEN UND LITERATUR}

Berbig, Roland / Krüger, Alexander: Ein Novum unter der Ägis eines Lehrstuhlinhabers. Walter Höllerer im Jahr 1959. In: Berliner Hefte zur Geschichte des literarischen Lebens, Bd. 8 (2008), S. 89-99.

Bogdal, Klaus-Michael: Klimawechsel. Eine kleine Meteorologie der Gegenwartsliteratur. In: Erb, Andreas (Hg.) unter Mitarbeit von H. Krauss u. J. Vogt: Bausteine Gegenwartsliteratur. Die neunziger Jahre. Opladen / Wiesbaden 1998, S. 9-31.

Böttiger, Helmut (unter Mitarbeit von Lutz Dittrich): Elefantenrunden.

Walter Höllerer und die Erfindung des Literaturbetriebs. Berlin 2005 (= Texte aus dem Literaturhaus Berlin, Bd. 15).

Briegleb, Klaus: 1968. Literatur in der antiautoritären Bewegung. Frankfurt am Main 1993 (es 1669).

Deglmann, Irmgard: Die Anfänge der Zeitschrift für Dichtung >Akzenteく. Magisterarbeit, Univ. München 1986 (im Literaturarchiv Sulzbach-Rosenheim).

Dirks, Walter: Heilige Allianz. Bemerkungen zur Diffamierung der Intellektuellen. In: Frankfurter Hefte (1961), S. 23-32.

Göpfert, Herbert G.: »Akzente« - vorgeschichtlich. In: Miller, Norbert / Klotz, Volker / Krüger, Michael (Hg.): Bausteine zu einer Poetik der Moderne. Festschrift für Walter Höllerer. München 1987, S. 99-105.

Handt, Friedrich [d.i. Höllerer, Walter] (Hg.): Deutsch - gefrorene Sprache in einem gefrorenen Land? Polemik Analysen Aufsätze. Berlin 1964.

Handt, Friedrich [d.i. Höllerer, Walter]: Das hohle Wunder. Bericht über drei Aufsätze von George Steiner, John McCormick und Hans Habe. In: Ders. (Hg.): Deutsch - gefrorene Sprache in einem gefrorenen Land? Polemik Analysen Aufsätze. Berlin 1964, S. 9-26.

Handt, Friedrich [d.i. Höllerer, Walter]: Nachwort. In: Ders. (Hg.): Deutsch - gefrorene Sprache in einem gefrorenen Land? Polemik Analysen Aufsätze. Berlin 1964, S. 201.

Höllerer, Walter (Hg.): Ein Gedicht und sein Autor. Lyrik und Essay. Berlin 1967.

Höllerer, Walter (Hg.): Transit. Lyrikbuch der Jahrhundertmitte. Frankfurt am Main 1956.

Höllerer, Walter: Die Elephantenuhr. Roman. Frankfurt am Main 1973. 
Höllerer, Walter: Erwiderung. In: Walter Höllerer zu Ehren. Festveranstaltung anlässlich der Verleihung der akademischen Würde Ehrenmitglied der Technischen Universität Berlin. Berlin, 12. Februar 1993 (TUB DOKUMENTATION. Kongresse und Tagungen, H. 62), S. 33-42.

Höllerer, Walter: Gedichte 1942-1982. Frankfurt am Main 1982.

Höllerer, Walter: Memorandum zur Gründung eines Instituts »Sprache im technischen Zeitalter« vom 8.12.1959. Abgedruckt in: Berliner Hefte zur Geschichte des literarischen Lebens, Bd. 8 (2008), S. 103-109.

Höllerer, Walter: Systeme. Neue Gedichte. Berlin 1969.

Höllerer, Walter: Vorlass Walter Höllerer. In: Literaturarchiv Sulzbach-Rosenberg, Bestandssignatur: 02WH.

Höllerer, Walter: Zur Sprache im technischen Zeitalter. In: Handt, Friedrich (Hg.): Deutsch - gefrorene Sprache in einem gefrorenen Land? Polemik Analysen Aufsätze. Berlin 1964, S. 186-200.

Jäger, Georg: Der Schriftsteller als Intellektueller. In: Hanuschek, Sven / Hörnigk, Therese / Malende, Christine (Hg.): Schriftsteller als Intellektuelle. Politik und Literatur im Kalten Krieg. Tübingen 2000 (Studien und Texte zur Sozialgeschichte der Literatur, Bd. 73), S. 1-25.

Kämper-van den Boogaart, Michael: »Und einmal muß es gesagt werden...« Der Autor und Germanist Walter Höllerer im Dienste der Gruppe 47. Ein Vorfall aus dem Jahr 1966. In: Zeitschrift für Germanistik 17 (2007), H. 1, S. 108-127.

Link, Jürgen: >Diskurs` und/oder >Ideologieく? In: kultuRRevolution. zeitschrift für angewandte diskurstheorie 4 (1983), S. 46-68.

Link, Jürgen: Das Gespenst der Ideologie. In: Baßler, Moritz / Gruber, Bettina / Wagner-Egelhaaf, Martina (Hg.): Gespenster. Erscheinungen, Medien, Theorien. Würzburg 2005, S. 335-347.

Link, Jürgen: Warum Foucault aufhörte, Symbole zu analysieren: Mutmaßungen über 〉Ideologie` und 〉Interdiskurs`. In: Dane, Gesa u.a. (Hg.): Anschlüsse. Versuche nach Michel Foucault. Tübingen 1985, S. 105-114.

Miller, Norbert / Klotz, Volker / Krüger, Michael (Hg.): Bausteine zu einer Poetik der Moderne. Festschrift für Walter Höllerer. München 1987.

Miller, Norbert: Laudatio auf Prof. Dr. phil. Walter Höllerer. »Serendipity: Über eine Methode, Literatur zu interpretieren«. In: Walter 
Höllerer zu Ehren. Festveranstaltung anlässlich der Verleihung der akademischen Würde Ehrenmitglied der Technischen Universität Berlin. Berlin, 12. Februar 1993 (= TUB DOKUMENTATION. Kongresse und Tagungen, H. 62), S. 19-32.

Miller, Norbert: Vorwort. In: Ders. / Klotz, Volker / Krüger, Michael (Hg.): Bausteine zu einer Poetik der Moderne. Festschrift für Walter Höllerer. München 1987, S. 7-10.

Neumann, Robert: Spezis. Gruppe 47 in Berlin. In: Konkret 5 (1966), S. 34-39.

Parr, Rolf / Thiele, Matthias: Link(s). Eine Bibliographie zu den Konzepten >Interdiskurs`, >Kollektivsymbolikı und >Normalismus` sowie einigen weiteren Fluchtlinien. 2., stark erw. und überarb. Aufl., Heidelberg 2010.

Parr, Rolf: >Sowohl als auch Status des Essays. In: Braungart, Wolfgang / Kauffmann, Kai (Hg.): Essayismus um 1900. Heidelberg 2006 (= Beihefte zum Euphorion. Zeitschrift für Literaturgeschichte, H. 50), S. 1-14.

Parr, Rolf: »Zwei Seelen wohnen, ach! in meiner Brust.« Strukturen und Funktionen der Mythisierung Bismarcks (1860-1918). München 1992.

Parr, Rolf: Paradoxe Medienästhetik. Martin Walsers >Realismus` und sein literarischer Umgang mit Film und Fernsehen. In: Geisenhanslüke, Achim / Mein, Georg / Schössler, Franziska (Hg.): Das Subjekt des Diskurses. Festschrift für Klaus-Michael Bogdal zum 60. Geburtstag. Heidelberg 2008, S. 303-320.

Pfammatter, René: Essay - Anspruch und Möglichkeit. Plädoyer für die Erkenntniskraft einer unwissenschaftlichen Darstellungsform. Hamburg 2002 (= Poetica. Schriften zur Literaturwissenschaft, Bd. 67).

Pisiotis, Ulrike: Rivalität und Bündnis. Zum Verhältnis von Wissenschaft und Literatur der Moderne. Dresden 2012 (= Kulturstudien, Bd. 7).

Richter, Hans Werner: Das Lachen aus der Oberpfalz. Walter Höllerer. In: Ders.: Im Etablissement der Schmetterlinge. Einundzwanzig Portraits aus der Gruppe 47. München 1986, S. 149-159.

Schlösser, Hermann: Literaturgeschichte und Theorie in der Literatur. In: Hansers Sozialgeschichte der deutschen Literatur vom 16. Jahrhundert bis zur Gegenwart. Hg. von Rolf Grimminger. Bd. 12: 
Gegenwartsliteratur seit 1968. Hg. von Klaus Briegleb und Sigrid Weigel. München 1992, S. 385-403.

Walter Höllerer zu Ehren. Festveranstaltung anlässlich der Verleihung der akademischen Würde Ehrenmitglied der Technischen Universität Berlin. Berlin, 12. Februar 1993 (= TUB DOKUMENTATION. Kongresse und Tagungen, H. 62).

Walter Höllerer. $\mathrm{Zu}$ seinen Gedichten und seiner Lyrik-Anthologie Transit. Briefe und Texte Band 1. Sulzbach-Rosenberg 2002.

Wellbery, David E. u.a. (Hg.): Eine neue Geschichte der deutschen Literatur. Berlin 2007. 
Dieser Text wird über DuEPublico, dem Dokumenten- und Publikationsserver der Universität Duisburg-Essen, zur Verfügung gestellt. Die hier veröffentlichte Version der EPublikation kann von einer eventuell ebenfalls veröffentlichten Verlagsversion abweichen.

DOI: $\quad 10.14361 /$ transcript.9783839415986.191

URN: urn:nbn:de:hbz:464-20210224-164842-4

Parr, Rolf: Walter Höllerers Neuakzentuierung der Intellektuellenrolle im Literaturbetrieb. DOI: https://doi.org/10.14361/transcript.9783839415986.191

In: Poetik im technischen Zeitalter : Walter Höllerer und die Entstehung des modernen Literaturbetriebs / Geisenhanslüke, Achim ; Hehl, Michael Peter (Hrsg.). - Bielefeld: transcript-Verlag, 2013 - eISBN 978-3-8394-1598-6.

DOI: https://doi.org/10.14361/transcript.9783839415986, S. 191-212

(C) 2013 transcript Verlag, Bielefeld. Alle Rechte vorbehalten. 